\title{
A convenient access to ring unsaturated cyclopentanecarbodithioates via hetero-Diels-Alder chemistry
}

\author{
Ibrahim El-Sayed,*a Rita Grønbæk Hazell, ${ }^{\mathrm{b}}$ and Alexander Senning*c \\ ${ }^{a}$ Chemistry Department, Faculty of Science, Menoufia University, Shebin El-Kom, Egypt \\ ${ }^{b}$ Department of Chemistry, Aarhus University, DK-8000 Århus C, Denmark, and ${ }^{c}$ Department of \\ Chemistry, Technical University of Denmark, DTU 207, DK-2800 Kgs. Lyngby, Denmark \\ E-mail: eltantawy_2000@yahoo.com; aes@kemi.dtu.dk
}

\section{Dedicated to Professor Boris A. Trofimov on the occasion of his $65^{\text {th }}$ birthday}

(received 02 Feb 03; accepted 28 Apr 03; published on the web 02 May 03)

\begin{abstract}
Chlorodithioformates react with cyclopenta-1,3-dienes to give the corresponding hetero-DielsAlder adducts which in turn eliminate $\mathrm{HCl}$ to form, depending on the substitution pattern, cyclopenta-2,4-dienecarbodithioates or cyclopent-2-enecarbodithioates with an additional exocyclic $\mathrm{C}=\mathrm{C}$ double bond. The key structures $\mathbf{5 b}$ and $\mathbf{5 c}$ were determined by $\mathrm{X}$-ray crystallography.
\end{abstract}

Keywords: Chlorodithioformates, cyclopenta-1,3-dienes, hetero-Diels-Alder reaction

\section{Introduction}

Our earlier review on chlorodithioformates, $\mathbf{1}$, could not report any examples of $\mathbf{1}$ acting as dienophiles, as opposed to their well-documented 1,3-dipolarophilic properties, ${ }^{1}$ and a somewhat earlier report from our laboratory explicitly stated that $\mathbf{1}$ (at ambient temperature) do not react with buta-1,3-diene. ${ }^{2}$ An attempted hetero-Diels-Alder reaction between trichloromethyl chlorodithioformate, $\mathrm{Cl}-\mathrm{CSSCCl}_{3}$, and anthracene led only to Friedel-Crafts substitution with no observable intermediate cycloadduct. ${ }^{3}$<smiles>[R]SC(=S)Cl</smiles>

1<smiles>S=C(Cl)SC(Cl)(Cl)Cl</smiles>

$1 a$<smiles>CCCCCC(C)(C)C(SC(=S)Cl)=C(c1ccccc1)c1ccccc1</smiles>

$1 b$

Scheme 1 
On the other hand, several closely related thiocarbonyl compounds such as thiophosgene, ${ }^{4}$ cyanodithioformates $\quad \mathrm{NC}-\mathrm{CSSR},{ }^{5} \quad C$-sulfonyldithioformates $\quad \mathrm{R}^{1} \mathrm{SO}_{2}-\mathrm{CSSR},{ }^{2,6}$ and phosphonyldithio-formates, $\left(\mathrm{R}^{1} \mathrm{O}\right){ }_{2} \mathrm{PO}-\mathrm{CSSR},{ }^{2,7}$ do exhibit a rich hetero-Diels-Alder chemistry with 1,3-dienes.

\section{Results and Discussion}

Following earlier work on hetero-Diels-Alder reactions of chlorodithioformates, including 1, with acyclic 1,3-dienes ${ }^{8}$ we have now found that the corresponding reactions of $\mathbf{1} \mathbf{a}^{9 \mathrm{a}}$ and $\mathbf{1} \mathbf{b}^{9 \mathrm{~b}}(c f$. Scheme 1) with the cyclopenta-1,3-dienes $2 \mathbf{a}-\mathbf{c}^{10}$ (cf. Scheme 2) are even easier, i.e., proceed at ambient temperature whereas the former required heating to $80^{\circ} \mathrm{C}$.

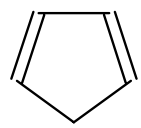

$2 a$

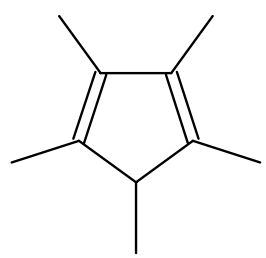

$2 b$

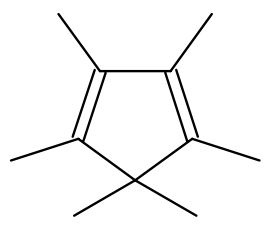

2c

\section{Scheme 2}

In all cases, the primary Diels-Alder adduct 3 cannot be observed, but is apparently subject to easy rearrangement to the monocyclic dithioformate 4 which, in turn, more or less readily loses hydrogen chloride and gives the quasi-Friedel-Crafts product 5, $c f$. Scheme 3 . In the case of 4a the spectroscopic data of the isolated product were satisfactory, while the results of the outof-house elemental analysis showed extensive, but not quantitative, conversion to 5a by loss of $\mathrm{HCl}$. The driving force for the rearrangement $\mathbf{3} \rightarrow \mathbf{6} \rightarrow \mathbf{4}$ (which is not observed with the corresponding Diels-Alder adducts from acyclic 1,3-dienes, ${ }^{8}$ nor with the thiophosgene-cyclopenta-1,3-diene cycloadduct ${ }^{4 a}$ ) is in all likelihood the heterolytic cleavage of an endocyclic C-S bond with formation of a zwitterion with a highly stabilized tertiary allylic carbocationic positive pole and a negative pole well-suited for the expulsion of an incipient chloride ion, Eqn. (1). The products 4a and/or 5a derived from $2 \mathbf{a}$ were difficult to purify and could not be fully characterized, while $\mathbf{5 b}$ and $5 \mathbf{c}$ were obtained in a high state of purity and characterized by the usual techniques, including single crystal X-ray crystallography. ${ }^{11}$ 
<smiles>FC(Cl)(Cl)C(F)(Cl)SC1(Cl)SC2C=CC1C2</smiles>

$3 a$<smiles>CC(F)(Cl)SC(=S)C1C=CC2CC21Cl</smiles>

$4 a$<smiles>CC(F)(Cl)SC(=S)C1C=CC=C1</smiles>

$5 a$

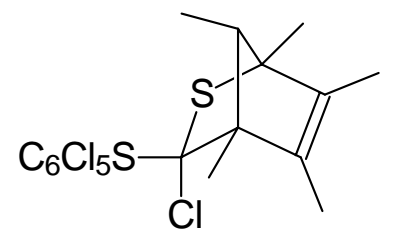

$3 b$<smiles>CC1=C(C)C2(Cl)C(C)(C(=S)SC(C)(C)C)C(=S)C1(C)C2(C)C</smiles>

$4 b$<smiles>CC1=C(C)C(C)(C(=S)SC(F)(F)F)C(C)=C1C</smiles>

$5 b$

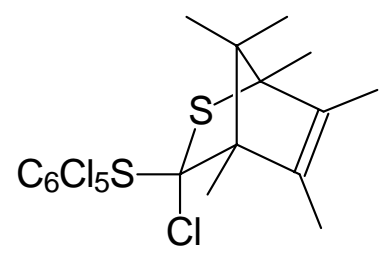

3c<smiles>CC1=C(C)C2(C)C(=S)C1(C)C(C)(C)C2(C)C(C)(C)[Ge]</smiles>

4c<smiles>C=C1C(C)=C(C)C(C)(C(=S)[Se]C(F)(F)Cl)C1(C)C</smiles>

5c

\section{Scheme 3}<smiles>CC1=C(C)C2(Cl)SC1(Cl)C(C)=C2C</smiles>

$3 b$

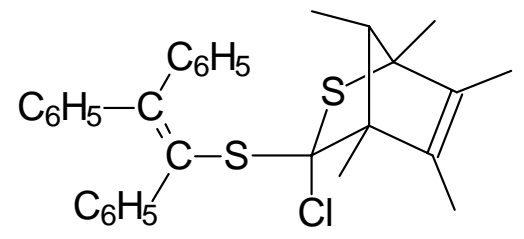

3d

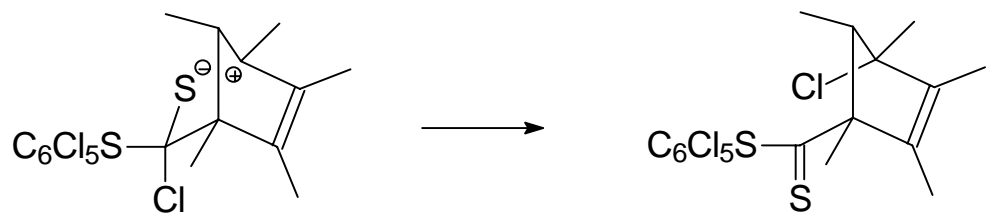

$4 b$<smiles>CC1=C(C)C2(Cl)C(=S)C1(C)C(SC([15CH])=C([15CH2])c1ccccc1)=C2C</smiles>

4d<smiles>CC1=C(C)C(C)(C(=S)SC(=C(C(C)(C)C)C(C)(C)C)C(C)(C)C)C(C)=C1C</smiles>

$5 d$

\section{Scheme 4}


Compound 5d was obtained in the same way, $c f$. Scheme 4. In the molecular structures the alkene $\mathrm{C}=\mathrm{C}$ bonds of $5 \mathbf{b}, \mathrm{C} 3-\mathrm{C} 4(1.344 \AA)$ and $\mathrm{C} 5-\mathrm{C} 6(1.348 \AA)$, and of 5c, C3-C4 (1.330 $\AA)$ and $\mathrm{C} 5-\mathrm{C} 10(1.337 \AA)$, are discernible as fully localized entities.

For recent work on the related Diels-Alder chemistry of spiro[2.4]hepta-4,6-diene and thiophosgene, see ref. $4 \mathrm{~b}$ No Diels-Alder reactions between thiocarbonyl compounds and $\mathbf{4 b}$ or $4 \mathbf{c}$ appear to have been recorded.

\section{Experimental Section}

General Procedures. All syntheses were carried out under an atmosphere of dry nitrogen. All NMR experiments (solvent $\mathrm{CDCl}_{3}$, TMS as internal standard) were carried out with a Varian Unity $400 \mathrm{MHz}$ spectrometer $\left(400 \mathrm{MHz}\right.$ for ${ }^{1} \mathrm{H}, 100 \mathrm{MHz}$ for ${ }^{13} \mathrm{C}$ ). The mass spectra were obtained in the EI mode (70 eV, direct inlet) with a Finnigan MAT GCQ PLUS system. The infrared spectra (KBr disks) were run on a Perkin-Elmer FT/IR-1760 spectrometer. The melting points were taken with an SMP3 apparatus.

1,2,3,4,5,5-Hexamethylcyclopenta-1,3-diene, (2c). Compound 2c was prepared by our improved version of an implied literature procedure. ${ }^{10}$ 1,2,3,4,5-Pentamethylcyclopenta-1,3dienyl anion was generated at $0{ }^{\circ} \mathrm{C}$ by treatment of 1,2,3,4,5-pentamethylcyclopenta-1,3-diene $(1.50 \mathrm{~mL}, 1.30 \mathrm{~g}, 9.54 \mathrm{mmol})$, dissolved in THF, with $9.54 \mathrm{~mL}(14.3 \mathrm{mmol})$ of $1.50 \mathrm{M}$ ethereal methyllithium/lithium bromide complex, and then treated with iodomethane $(1.78 \mathrm{~mL}, 4.06 \mathrm{~g}$, $28.6 \mathrm{mmol})$. The stirred reaction mixture was allowed to warm to room temperature $(1 \mathrm{~h})$, filtered, the solid residue extracted with ether, and the combined ether phases evaporated in vacuo. Subsequent Kugelrohr distillation of the oily residue at $32-34{ }^{\circ} \mathrm{C} / 0.55 \mathrm{~mm} \mathrm{Hg}$ afforded 2c as a colorless oil, yield $1.27 \mathrm{~g}(89 \%) .{ }^{1} \mathrm{H}$ NMR $\left(\mathrm{CDCl}_{3}\right): \delta 0.91$ (s, 6H, 5,5- $\left.\mathrm{Me}_{2}\right), 1.76(\mathrm{~s}, 6 \mathrm{H}$,

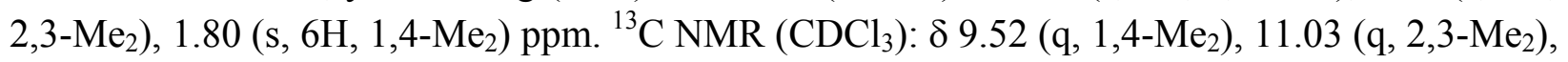
21.81 (q, 5,5- $\mathrm{Me}_{2}$ ), 52.14 (s, C-5), 131.85 (s, C-2, C-3), 142.32 (s, C-1, C-4), the assignments, where there could be doubt, are in accord with an ACD simulation. Courtneidge et al. ${ }^{10}$ report a peak at $138.08 \mathrm{ppm}$ in their ${ }^{13} \mathrm{C}$ NMR spectrum instead of our $131.85 \mathrm{ppm}$ peak-apparently a misprint in their paper. Our ACD/C-NMR simulation shows the corresponding peak at 131.89 ppm.

4-Chlorocyclopent-2-enecarbodithioic acid pentachlorophenyl ester, (4a). Chlorodithioformate 1a $(150 \mathrm{mg}, 0.41 \mathrm{mmol})$ was dissolved in $3 \mathrm{~mL}$ trichloromethane and treated with $0.14 \mathrm{~mL}$ (2.05 $\mathrm{mmol}$ ) of freshly distilled cyclopenta-1,3-diene. After $3 \mathrm{~h}$ all $\mathbf{1 a}$ had been consumed and, after removal of the solvent and addition of ether $(2 \mathrm{~mL})$ and cooling to $-10{ }^{\circ} \mathrm{C}$ a yellow solid precipitated which was washed three times with pentane to afford $139 \mathrm{mg}(86 \%)$ of 4 a as yellow crystals, m.p. 119-122 ${ }^{\circ} \mathrm{C}$. (Found: C, 35.95; H 1.71; Cl, 47.26; S, 14.51. Calculated for $\mathrm{C}_{12} \mathrm{H}_{6} \mathrm{Cl}_{6} \mathrm{~S}_{2}$, i.e., 4a: C, 33.75; H, 1.42; Cl, 49.81; S, 15.02\%: 427.02. Calculated for $\mathrm{C}_{12} \mathrm{H}_{5} \mathrm{Cl}_{5} \mathrm{~S}_{2}$, i.e., 5a: C, 36.90; H, 1.29; Cl, 45.39; S, 16.42\%: 390.56). Apparently, the analytical sample had 
lost some $\mathrm{HCl}$ while in transit. IR (KBr): v 3083, 2987, 2933, 1605, 1403, 1322, 1125, 833, 706, $687 \mathrm{~cm}^{-1} ;{ }^{1} \mathrm{H}$ NMR $\left(\mathrm{CDCl}_{3}\right): \delta 2.75(\mathrm{~m}, 2 \mathrm{H}), 4.79(\mathrm{~m}, 1 \mathrm{H}), 5.15(\mathrm{~m}, 1 \mathrm{H}), 6.15(\mathrm{~m}, 2 \mathrm{H}) ;{ }^{13} \mathrm{C}$ NMR (CDCl $\left.)_{3}\right): \delta 43.68(\mathrm{t}), 63.44(\mathrm{~d}), 63.48(\mathrm{~d}), 132.80$ (s), 133.88 (s), 135.44 (d), 135.98 (d), 136.78 (s), 138.34 (s), 230.61 (s); MS (EI): m/z (\%) 388 (45, M-HCl), 353 (100, $\mathrm{C}_{12} \mathrm{H}_{5} \mathrm{Cl}_{4} \mathrm{~S}_{2}$ ), $323\left(16, \mathrm{C}_{7} \mathrm{Cl}_{5} \mathrm{~S}_{2}\right), 246\left(18, \mathrm{C}_{6} \mathrm{Cl}_{5}\right), 212\left(60, \mathrm{C}_{6} \mathrm{Cl}_{4}\right), 109\left(40, \mathrm{C}_{6} \mathrm{H}_{5} \mathrm{~S}\right), 65\left(23, \mathrm{C}_{5} \mathrm{H}_{5}\right)$.

1,2,3,4,5-Pentamethylcyclopenta-2,4-dienecarbodithioic acid pentachlorophenyl ester, (5b). To a stirred solution of $\mathbf{1 a}(0.30 \mathrm{~g}, 0.83 \mathrm{mmol})$ in $10 \mathrm{~mL}$ trichloromethane was added $\mathbf{2 b}(0.26$ $\mathrm{mL}, 0.23 \mathrm{~g}, 1.69 \mathrm{mmol})$. The reaction mixture was stirred at room temperature for $45 \mathrm{~min}$. Evaporation in vacuo left crude $\mathbf{5 b}$ as an orange solid. Recrystallization from ether/hexane (1:3) yielded $0.35 \mathrm{~g}(92 \%)$ of $\mathbf{5 b}$ as orange crystals, m.p. $156-158{ }^{\circ} \mathrm{C}$. (Found: C, 43.90; H, 3.00; S, 14.60. Calculated for $\mathrm{C}_{17} \mathrm{H}_{15} \mathrm{Cl}_{5} \mathrm{~S}_{2}$ : C, 44.32; H, 3.28; S, 13.92\%: 460.704). IR (KBr): v 2961, 2909, 2852, 1652, 1435, 1338, 1303, $1274 \mathrm{~cm}^{-1} .{ }^{1} \mathrm{H}$ NMR $\left(\mathrm{CDCl}_{3}\right): \delta 1.39(\mathrm{~s}, 3 \mathrm{H}), 1.80(\mathrm{~s}, 6 \mathrm{H})$, 1.92 (s, 6H) ppm. ${ }^{13} \mathrm{C}$ NMR $\left(\mathrm{CDCl}_{3}\right): \delta 10.15$ (q), 11.63 (q), 21.30 (q), 75.03 (s), 132.24 (s), 132.55 (s), 135.67 (s), 138.83 (s), 139.50 (s), 139.68 (s), 234.22 (s). MS (EI): m/z (\%) 458 (12, $\left.\mathrm{M}, \mathrm{C}_{17} \mathrm{H}_{15} \mathrm{Cl}_{5} \mathrm{~S}_{2}\right), 423$ (100, $\left.\mathrm{C}_{17} \mathrm{H}_{15} \mathrm{Cl}_{4} \mathrm{~S}_{2}\right), 291$ (11, $\left.\mathrm{C}_{7} \mathrm{Cl}_{5} \mathrm{~S}\right), 134$ (29, $\left.\mathrm{C}_{10} \mathrm{H}_{14}\right), 119$ (24, $\left.\mathrm{C}_{9} \mathrm{H}_{11}\right)$, $91\left(14, \mathrm{C}_{7} \mathrm{H}_{7}\right)$.

\section{1,2,3,5,5-Pentamethyl-4-methylenecyclopent-2-enecarbodithioic acid pentachlorophenyl} ester (5c). To a stirred solution of $1 \mathbf{a}(0.30 \mathrm{~g}, 0.83 \mathrm{mmol})$ in $10 \mathrm{~mL}$ trichloromethane was added $0.25 \mathrm{~g}(1.66 \mathrm{mmol})$ of 2c. The reaction mixture was stirred at room temperature for 2 days. The solvent was then evaporated in vacuo and the residue purified by column chromatography (ether/pentane, 1:5) to afford an orange solid. Recrystallization from acetonitrile yielded $5 \mathbf{c}(0.28$ g, 72\%) as orange crystals, m.p. $129-132{ }^{\circ} \mathrm{C}$. (Found: C, 45.60; H 3.50; S, 13.30. Calculated for $\mathrm{C}_{18} \mathrm{H}_{17} \mathrm{Cl}_{5} \mathrm{~S}_{2}$ : C, 45.54; H, 3.60; S, 13.50\%: 474.731). IR (KBr): v 3082, 2976, 2937, 2914, 1627 , 1516, 1449, 1380, 1339, 1307, 1121, 1100, 866, 773, $693 \mathrm{~cm}^{-1} ;{ }^{1} \mathrm{H}$ NMR $\left(\mathrm{CDCl}_{3}\right.$, NOESY): $\delta$ $1.08\left(\mathrm{~s}, 3 \mathrm{H}, 5-\mathrm{Me}_{\mathrm{a}}\right), 1.14\left(\mathrm{~s}, 3 \mathrm{H}, 5-\mathrm{Me}_{\mathrm{b}}\right), 1.44$ (s, 3H, 1-Me), 1.84 (tq, 3H, 2-Me, ${ }^{6} \mathrm{~J}=0.66 \mathrm{~Hz},{ }^{5} \mathrm{~J}$ $=0.96 \mathrm{~Hz}), 1.91\left(\mathrm{q}, 3 \mathrm{H}, 3-\mathrm{Me},{ }^{5} \mathrm{~J}=0.96 \mathrm{~Hz}\right), 4.75\left(\mathrm{dq}, 1 \mathrm{H},=\mathrm{CH}_{\mathrm{a}},{ }^{2} \mathrm{~J}=0.40 \mathrm{~Hz},{ }^{6} \mathrm{~J}=0.66 \mathrm{~Hz}\right)$, $4.92\left(\mathrm{dq}, 1 \mathrm{H},=\mathrm{CH}_{\mathrm{b}},{ }^{2} \mathrm{~J}=0.40 \mathrm{~Hz},{ }^{6} \mathrm{~J}=0.66 \mathrm{~Hz}\right) \mathrm{ppm} ;{ }^{13} \mathrm{C} \mathrm{NMR}\left(\mathrm{CDCl}_{3}\right): \delta 10.61$ (q), $12.34(\mathrm{q})$, 21.52 (q), 22.78 (q), 30.35 (q), 49.21 (s), 74.34 (s), 99.86 (dd), 132.29 (s), 132.47 (s), 133.43 (s), 135.67 (s), 138.33 (s), 144.15 (s), 162.28 (s), 238.73 (s) ppm; MS (EI): m/z 472 (71, M, $\left.\mathrm{C}_{18} \mathrm{H}_{17} \mathrm{Cl}_{5} \mathrm{~S}_{2}\right), 323$ (37, $\left.\mathrm{C}_{7} \mathrm{Cl}_{5} \mathrm{~S}_{2}\right), 279\left(4, \mathrm{C}_{6} \mathrm{Cl}_{5} \mathrm{~S}\right), 149\left(100, \mathrm{C}_{11} \mathrm{H}_{17}\right)$.

1,2,3,4,5-Pentamethylcyclopenta-2,4-dienecarbodithioic acid 1,2,2-triphenylethenyl ester (5d). To a stirred solution of $\mathbf{1 b}(0.20 \mathrm{~g}, 0.54 \mathrm{mmol})$ in $5 \mathrm{~mL}$ trichloromethane was added $0.15 \mathrm{~g}$ $(1.10 \mathrm{mmol})$ of $\mathbf{2 b}$. The reaction mixture was stirred at room temperature for $1 \mathrm{~h}$. It was then evaporated in vacuo and the orange residue recrystallized from hexane to yield $5 \mathbf{d}(0.22 \mathrm{~g}, 87 \%)$ as orange crystals, m.p. $132-135{ }^{\circ} \mathrm{C}$. (Found: $\mathrm{C}, 78.79 ; \mathrm{H}$ 6.66; S, 13.13. Calculated for $\mathrm{C}_{31} \mathrm{H}_{30} \mathrm{~S}_{2}: \mathrm{C}, 79.78 ; \mathrm{H}, 6.48 ; \mathrm{S}, 13.74 \%$ : 466.712). IR (KBr): v 2967, 2924, 2870, 1599, 1444, 1378, 1074, 926, 745, $698 \mathrm{~cm}^{-1} ;{ }^{1} \mathrm{H} \mathrm{NMR}\left(\mathrm{CDCl}_{3}\right): \delta 1.24(\mathrm{~s}, 3 \mathrm{H}), 1.41(\mathrm{~s}, 6 \mathrm{H}), 1.78(\mathrm{~s}, 6 \mathrm{H})$, 6.96-7.27 (m, 15H) ppm; ${ }^{13} \mathrm{C}$ NMR $\left(\mathrm{CDCl}_{3}\right): \delta 9.93$ (q), 11.45 (q), 21.13 (q), 74.52 (s), 126.70 (d), 126.90 (d), 127.20 (d), 127.51 (d), 127.55 (d), 128.01 (d), 128.70 (d), 130.80 (d), 131.15 (d), 131.40 (s), 138.43 (s), 139.28 (s), 139.45 (s), 141.77 (s), 143.05 (s), 152.31 (s), 237.72 (s, C=S) 
ppm; MS (EI): m/z (\%) $466\left(59, \mathrm{M}, \mathrm{C}_{31} \mathrm{H}_{30} \mathrm{~S}_{2}\right), 255$ (100, $\left.\mathrm{C}_{20} \mathrm{H}_{15}\right), 179\left(31, \mathrm{C}_{11} \mathrm{H}_{15} \mathrm{~S}\right), 135$ (83, $\left.\mathrm{C}_{10} \mathrm{H}_{15}\right)$.

X-Ray crystallographic study of 1,2,3,4,5-pentamethylcyclopenta-2,4-dienecarbodithioic acid pentachlorophenyl ester (5b). Crystal data: crystal system, monoclinic; space group, $\mathrm{P} 2{ }_{1} / \mathrm{c} ; a=8.6993(5) \AA ; b=29.353(2) \AA ; c=8.6614(5) \AA ; \beta=117.790(1)^{\circ} ; \mathrm{V}=1956.6(2) \AA^{3} ; \mathrm{Z}$ $=4$; crystal size $0.37 \times 0.26 \times 0.09 \mathrm{~mm}$; linear absorption coefficient $0.951 \mathrm{~mm}^{-1}$; transmission factors $0.919-0.678$; total number of unique reflections $5872 ;[\mathrm{I}>2 \sigma(\mathrm{I})]=3738$; $\theta$-range $2.7-30.3^{\circ} ; \mathrm{R}($ obs. data) $=0.075 ; \mathrm{wR} 2$ (all data) $=0.062$.

X-Ray crystallographic study of 1,2,3,5,5-pentamethyl-4-methylenecyclopent-2enecarbodithioic acid pentachlorophenyl ester (5c). Crystal data: crystal system, monoclinic; space group, $\mathrm{P} 2{ }_{1} / \mathrm{c} ; a=10.761(1) \AA ; b=11.475(2) \AA ; c=16.846(2) \AA ; \beta=94.015(3)^{\circ} ; \mathrm{V}=$ 2075.1(5) $\AA^{3} ; Z=4$; crystal size $0.56 \times 0.23 \times 0.18 \mathrm{~mm}$; linear absorption coefficient $0.90 \mathrm{~mm}^{-1}$; transmission factors $0.893-0.662$; total number of unique reflections 6390 ; $[\mathrm{I}>2 \sigma(\mathrm{I})]=5025$; $\theta$ range $2.4-30.5^{\circ} ; \mathrm{R}$ (obs. data) $=0.050 ; \mathrm{wR} 2$ (all data) $=0.056$.

The two structures are not of high quality, despite being measured at $120 \mathrm{~K}$, because of lack of strong packing forces.

\section{Acknowledgments}

Partial financial support by DANIDA (Danish International Development Assistance) is gratefully acknowledged.

\section{References and Notes}

1. El-Sayed, I.; Abdel-Megeed, M. F.; Yassin, S. M.; Senning, A. Sulfur Rep. 1995, 16, 235.

2. Boerma, J. A.; Nilsson, N. H.; Senning, A. Tetrahedron 1974, 30, 2735.

3. Moltzen, K. E.; Senning, A.; Kramer, M. P.; Klabunde, K. J. J. Org. Chem. 1984, 49, 3854.

4. (a) Middleton, W. J. J. Org. Chem. 1965, 30, 1390. (b) Föhlisch, B.; Bakr, D. A.; Fischer, P. J. Org. Chem. 2002, 67, 3682.

5. Vyas, D. M.; Hay, W. Can. J. Chem. 1975, 53, 1362.

6. Yassin, S. M. Sulfur Rep. 1995, 16, 343.

7. Heuzé, B.; Gasparova, R.; Herras, M.; Masson, M. Tetrahedron Lett. 2000, 41, 7327.

8. El-Sayed, I.; Senning, A. Sulfur Lett. 2002, 25, 263.

9. (a) Hansen, H. C.; Jensen, B.; Senning, A. Sulfur Lett. 1985, 3, 61. (b) El-Sayed, I.; AbdelMegeed, M. F.; Yassin, S. M.; Senning, A. Phosphorus, Sulfur, Silicon Relat. Elem. 1994, 86, 239.

10. Courtneidge, J. L.; Davies, A. G.; Shields, C. J.; Yazdi, S. N. J. Chem. Soc., Perkin Trans. 2 1988, 799 and references cited therein. 
11. The full crystallographic data for $\mathbf{5 b}$ and 5c have been deposited with the Cambridge Crystallographic Data Centre, reference number CCDC 191808 (5b) and CCDC 191809 (5c). Copies of the data can be obtained on application to CCDC, 12 Union Road, Cambridge CB2 1EZ, UK (E-mail: deposit@ccdc.cam.ac.uk). 